\title{
Pitfalls in Comparing Functional Magnetic Resonance Imaging and Invasive Electrophysiology Recordings
}

\author{
DLG Hill ${ }^{1}$, A Simmons ${ }^{23}$, AD Castellano Smith ${ }^{1}, \mathrm{CR}$ Maurer, $\mathrm{Jr}^{1}{ }^{1}, \mathrm{TCS}_{\mathrm{Cox}}{ }^{12}$, \\ R Elwes ${ }^{2}$, MJ Brammer ${ }^{3}$, DJ Hawkes ${ }^{1}$, CE Polkey $^{2}$ \\ ${ }^{1}$ Radiological Sciences \& Medical Engineering, ${ }^{2}$ Clinical Neurosciences and ${ }^{3}$ Institute of \\ Psychiatry, King's College London, UK. <D.Hill@umds . ac . uk>
}

\begin{abstract}
Several authors have recently compared the results of fMRI studies on neurosurgery patients with invasive electrophysiology. These studies aim to validate fMRI against an accepted gold standard, and ascertain whether fMRI could replace invasive electrophysiology in neurosurgical patients. We have identified and quantified two characteristics of these data that make such comparisons problematic. Firstly, the epilepsy surgery patients $(n=8)$ studied move significantly more during fMRI experiments than normal volunteers $(n=6)$ performing the same task. This motion has a particularly large out-of-plane component, and is significantly more correlated with the stimulus than for the normal volunteers. This motion is especially large when performing a task on the side affected by the lesion. This additional motion is hard to correct and substantially degrades the quality of the resulting fMRI images, making it a much less reliable technique on these surgical patients than on other subjects. Secondly, we have found that, following electrode implantation, the brain surface can shift by more than $10 \mathrm{~mm}$ relative to the skull compared to its preoperative location, substantially degrading the accuracy of the comparison of electrophysiology measurements made on the deformed brain and $\mathrm{AMRI}$ studies carried out preoperatively. Taken together, these findings suggest that studies of this sort are currently of limited use for validating fMRI, and further image analysis research is necessary to solve the problems caused by subject motion and brain deformation.
\end{abstract}

\section{Introduction}

Several authors have recently compared the results of functional Magnetic Resonance imaging (fMRI) studies on neurosurgery patients with subsequent electrophysiology studies carried out using chronically implanted sub-dural electrodes or intraoperative evoked potentials and stimulation [1-5]. These studies, which are reviewed below, contribute to an understanding of the relationship between functional regions localised using fMRI and the same regions localised using invasive electrophysiology. They do not, however, demonstrate that fMRI is sufficiently accurate and sensitive to replace invasive cortical mapping in surgery planning. Neither do they accurately validate fMRI against the established alternative technique of invasive cortical mapping. The results described in the literature tend to be qualitative, for example concluding that there is "good agreement" between the techniques, without stating what constitutes good agreement. Where quantitative data is provided, the accuracy of the measurements (eg: from aligning preoperative images with intraoperative photographs) is unclear.

We have carried out a similar study of epilepsy surgery patients, and noticed several features of our data that have important consequences for studies of this type. In particular, during fMRI, the neurosurgical epilepsy patients move more than volunteers and 
other patients studied at our institution, which affects the quality of the fMRI results. Furthermore, there is a clear shift in the position of the brain surface between preoperative MR panel and post-implantation CT scans. This brain deformation has implications for the position of sites in the brain identified during the two measurements, and any comparison of their location. We have quantified both the subject motion and brain deformation on a series of eight patients.

\subsection{Review of literature}

Jack's paper [1] states two benefits of this sort of study: to assess the suitability of fMRI as a tool for "localization of functional areas relative to the surgical target", and "validation of the physiologic truth of functional localization with MR imaging... with the accepted criterion standard of invasive cortical mapping". Two epilepsy surgery cases were studied. Both had $\mathrm{fMRI}$ and intraoperative electrode recordings (evoked potentials and median nerve stimulation). The first patient additionally had measurements taken using chronically implanted subdural electrode strips. For patient 1 , two volume renderings of the brain surface were generated. The first shows the relationship between the brain surface, the tumour boundary and the orientation of the single fMRI slice. The second showed the position of the electrodes and tumour overlaid on the rendered MR brain surface. The electrodes were localised using a post-implantation CT scan registered to the preoperative MR. Visual comparison of the two renderings "confirmed the impression formed from the functional MR study that the tumour straddled the functional sensorimotor strip". The results from the second patient were more problematic due to motion during fMRI imaging, but once again, the electrode measurements "confirmed the impression formed at functional MR imaging".

Yousry et al [2] share the objectives of Jack et al. They studied six patients and four volunteers. All the patients had space occupying lesions close to the pre-central gyrus, and one had a slight palsy. All patients and volunteers underwent an fMRI examination while carrying out a motor task. Electrophysiological measurements were made on the patients using intraoperative stimulation under local or balanced anaesthesia. The patients were administered corticosteroids and diuretics before craniotomy, so "there was no detectable brain displacement after opening the dura." Functional regions identified during stimulation were marked with small numbered labels placed on the brain surface, and the positions of the labels was recorded using an intraoperative photograph and sketch. In order to compare the fMRI and electrophysiology results, they first defined the motor hand area from the fMRI as the parenchymal area where the motor task resulted in a temporally correlated statistically significant regional signal increase. The corresponding functional region was defined intraoperatively as the area where electrical cortical stimulation of the central region elicited compound muscle action potentials from the contralateral hypothenar or thenar muscle, or a tonic opening or closing of the contralateral hand. Comparison of the locations of the functional region identified in these ways was achieved by measuring the distance between the target point and each of four anatomical landmarks (lines) visible in both the MR image and intraoperatively. The precise nature of the coordinate systems defined by these landmarks is unclear from the paper. The discrepancy in the measurements varied from -3 to $10 \mathrm{~mm}$. A Wilcoxon test was used to assess the significance of these discrepancies. The resulting $\mathrm{P}$ value 
was greater than 0.5 , from which the authors appear to have concluded that the two sets of measurements agreed. No study of the reproducibility of the measurements was reported. Yousry et al additionally found that the activated region in the fMRI was more diffuse in the patients than for the normal volunteers.

Puce et al [3] studied 4 patients ( 3 with focal epilepsy, 1 with intractable posttraumatic seizures) and six normal volunteers. For both groups, fMRI images were acquired using both dual slice conventional gradient echo and three-slice gradient-echo EPI, with motor and sensory hand activation tasks. For all patients, electrophysiological measurements were made using intraoperative cortical stimulation. For one patient, measurements were also made using chronically implanted subdural electrodes. Intraoperative photographs were taken using a camera mounted above the surgical field. A photograph was first taken of the bare brain, then with a recording grid in place, and also of the brain together with numerical labels placed on the brain surface to indicate sites of positive cortical stimulation. The coordinates of these sites were transferred onto an image of the rendered brain surface obtained from a segmented gradient echo MR volume. There is no consideration of errors introduced by the perspective geometry of the photograph compared to the rendering. For the patient who underwent chronic recordings, a post implantation MR volume was acquired to localize the electrodes. The fMRI was aligned with the anatomical gradient echo MR volume using an unspecified registration algorithm. Inspection of the renderings and comparison with the photographs lead them to report that "the delineation of hand sensorimotor cortex using functional MR imaging and two electrophysiological methods revealed good agreement on the location of sensory and motor areas". They conclude that "the correspondence of the functional MR imaging and electrophysiological maps, however, argues strongly for the accurate localization of the hand sensorimotor cortex by our MR imaging methods". No quantitative assessment of the discrepancy between the location of functional regions by the two methods is given.

Yetkin et al [4] study a relatively large series of 28 patients ( 22 with chronic epilepsy, 5 with a cerebral tumour, and one with an AVM). Their primary aim is to validate fMRI. These subjects carried out two tasks during fMRI imaging, word generation and finger tapping. Seven $1 \mathrm{~cm}$ thick sagittal EPI slices were acquired at a rate of 1 per second during the activation paradigm. All subjects underwent intraoperative stimulation mapping under local anesthesia. Each subject's hand, fingers, face and tongue were monitored during the stimulation and that subject counted or recited sentences while the surgeon searched for a region which, when stimulated, resulted in reproducible interruption of speech. Labels were placed on the brain at each stimulation site, and the marker placement was recorded photographically using a camera "positioned so as to minimize parallax and distortion". The fMRI and electrophysiological location of the functional regions were compared by aligning the most superficial slice of the fMRI showing activation with the digitized intraoperative photograph using Adobe Photoshop to scale, rotate and translate the images. Anatomical landmarks in the region were used as cues in performing this alignment. The discrepancies in the location of the corresponding functional regions in the two modalities were quantified by measuring the in-plane distance between the centre of the cortical stimulation site and the centre of the corresponding region activated in fMRI. Where there was no continuous region ac- 
tivated in fMRI, the distance between the centre of the cortical stimulation site and the closest activated pixel in the fMRI was measured instead. In all cases, the in-plane discrepancy was less than $20 \mathrm{~mm}$, and for $86 \%$ of measurement it was within $10 \mathrm{~mm}$. There is no estimate of out-of-plane discrepancy in location, or of errors in the registration of a single slice of fMRI to the photograph. They estimated the distortion in the EPI to reach one pixel and the precision of the fMRI to be $5 \mathrm{~mm}$ from reproducibility studies. They conclude that, despite the significant methodological problems, functional MR imaging represents a non-invasive means of anticipating the results of cortical mapping.

Fitzgerald et al [5] studied 11 patients with a lesion in the presumed dominant hemisphere, 8 with tumours, 1 with a cyst, 1 with epilepsy and one with a cavernous angioma. Their aim was to evaluate fMRI as a predictive technique for locating eloquent areas in the dominant hemisphere, and in particular, its accuracy at locating language areas for planning surgical resection. They also stated that the study had implications for validating fMRI on normal volunteers. All subjects underwent fMRI examinations using multi-slice spin-echo EPI, while performing one or more language tasks. Cortical stimulation was carried out intraoperatively with the patient under local anesthesia. Tags were placed on the brain surface to mark language areas, and an intraoperative photograph was used to record the location of the tags. A volumetric gradient echo MR image from each patient was registered to an MR angiogram using Analyze, and a rendering produced showing the brain surface and blood vessel landmarks. The statistical fMRI map was registered to the rendering in an unspecified way, and cortical activity could then be projected onto the surface of the brain. The lateral projection of the rendering was aligned with the intraoperative photograph using Adobe Photoshop to scale, translate and rotate the images until the user considered the position of the landmarks in the photo and in the rendering to be in "good agreement". The authors estimated the error in this process to be less than $7 \mathrm{~mm}$ for all patients, with $1 \mathrm{~mm}$ matching accuracy achieved for four patients. Agreement between the modalities was assessed by counting the number of activation areas in fMRI that contacted, overlapped or surrounded a language tags in the photograph. They additionally measured the distance between the centre of the region of activation in the fMRI and the language tags (it is not clear whether these measurements were carried out in the plane of the overlay, or in three dimensions). Sensitivity was considered to be the percentage of matches between fMRI regions and language tags, and specificity was the percentage of regions activated in fMRI that matched with a non-language tag. Depending on the stringency of the match criterion, the sensitivity across all subjects varied from $81 \%$ with a specificity of $51 \%$ to $92 \%$, with a specificity of $0 \%$.

\section{Method}

\subsection{Data Acquisition}

Eight patients with intractable epilepsy ( 4 of whom were judged clinically to have a motor deficit), were studied. Preoperatively, each patient was imaged using a 3D spoiled GRASS sequence and functional MRI on a 1.5T GE Signa MR system retrofitted with Advanced NMR hardware and software. For each study, $100 \mathrm{~T} 2 *$ weighted multi-slice datasets $(\mathrm{TE}=40 \mathrm{~ms}, \mathrm{TR}=3 \mathrm{~s}, 128 \times 64$ acquisition matrix, $3.1 \times 3.1 \times 5 \mathrm{~mm}$ voxels, $0.5 \mathrm{~mm}$ 
slice gap, 10 slices) were acquired using gradient echo EPI. We refer to each multi-slice dataset as a frame. Rest and activation epochs are alternated for 30 second periods over a total imaging time of five minutes. Activation experiments were performed for both hands using a finger opposition task. Head movement was limited by foam padding within the head coil and a restraining band across the forehead. Daily quality assurance was carried out to ensure high signal to ghost ratio, high signal to noise ratio and excellent temporal stability using an automated quality control procedure [6]. Each patient subsequently had surgery to implant one or more subdural electrode mats, varying in size from 8 to 64 electrodes per mat, on a $1 \mathrm{~cm}$ grid. All patients in this study had at least 32 electrodes implanted. During the days following implantation, electrophysiological measurements were carried out to localize the epileptogenic zone, and eloquent regions of the cortex in the vicinity of the site of surgery. Immediately after implantation of the mat, or immediately prior to removal, the patient had a neuro-CT scan. Subsequent resection was planned in the normal way, without taking into account the fMRI data. Six normal volunteers under-went the same fMRI paradigm.

\subsection{Data Processing}

For each fMRI study, all 100 frames were registered to the first frame using an algorithm that minimises the mean square difference between voxel intensities [7]. This algorithm produces a motion trajectory for each subject showing movement during the scan for six degrees of freedom (three translations and three rotations). Subject motion was quantified using two indices for each degree of freedom: the range of motion; and the value of the power spectrum of the motion at the stimulation frequency. The second index measures how correlated the motion is with the stimulus. The indices were used to compare the patients with the volunteers, and also to compare the amount of subject motion when a patient was carrying out a task using the side of the brain affected by the lesion with the contralateral side. Subsequent fMRI processing was carried out using the algorithm of Bullmore et al [8].

Each patient's gradient echo MR volume was registered to the post-implantation CT scan using an algorithm that optimises the mutual information of the joint probability distribution of the two images [9]. This algorithm was found in an international multicentre trial to have a median registration error of less than $2 \mathrm{~mm}$ for retrospective MR$\mathrm{CT}$ registration [10]. The MR and CT images being registered in the current study are different from those in the multi-centre study (eg: the CT scans contain high contrast structures such as electrodes and air within the head that were not present in the MR), but this did not cause the algorithm to fail, and visual assessment of the results suggested the accuracy was similar [11]. For all patients in this study, the electrode mat is clearly visible in the post-implantation CT scan lying some distance medial to the inner table of the skull. The region between the mat and the skull is filled with air and fluid. If the $\mathrm{CT}$ scan is thresholded to show only bone and electrodes and overlaid on the registered MR volume, the electrodes are seen to lie several $\mathrm{mm}$ inside the MR brain surface. We believe that this shift in position is due to deformation of the brain tissue caused by the surgical procedure to open the dura and implant the electrodes. We quantified the magnitude of this brain deformation in two ways: by measuring the displacement between each electrode on the mat and nearest point on the inner table of the skull, and 
secondly by measuring the distance between each electrode on the mat and the envelope of the brain surface. We identified the 3D coordinates of the electrodes in the mat using a cursor in surface renderings of the CT scans. The rendering is a parallel projection in the sagittal plane with a z-buffer that stores the distance to the first voxel with an intensity over a specified threshold. The two dimensional coordinates of the cursor on the rendering can therefore be converted to three dimensional coordinates in the postimplantation CT scan using the z-buffer depth information. By rendering both sides of the brain, it is possible to identify the inner and outer surfaces of the electrodes (which are about $1 \mathrm{~mm}$ thick). The Analyze software package (Biomedical Imaging Resource, Mayo Clinic, Rochester MN) was used to automatically trace the inner table of the skull from the CT scan, and also to manually trace the envelope of the brain surface from the MR volume. The brain envelope is an estimate of where the electrode mat might lie in MR coordinates in the absence of brain deformation. The envelope does not follow the convoluted surface of the cortex, as the electrode mat is relatively stiff, and cannot, therefore penetrate sulci. Also, the envelope is not the same as the dura surface, as in some of our patients, there was atrophy in the region where the mat was placed, so the tops of the gyri are separated from the dura by CSF. The rigid body transformation found by registering the MR and CT images was then used to transform the brain envelope surfaces into the same coordinate frame as the CT electrodes and inner surface of the skull. The skull and brain surfaces were then triangulated using the Nuages software package [12]. The Euclidean distance between each electrode and the closest facet in the two surfaces was calculated.

\section{Results}

In our neuroimaging unit, we regularly produce high quality fMRI images from normal volunteers and non-surgical patients (eg: [13-15]). The fMRI images from the epilepsy surgery patients presented in this paper were, however, visually judged to be of much lower quality, probably because of motion.

\begin{tabular}{|l|c|c|}
\hline degree of freedom & volunteer & patient \\
\hline $\mathrm{x}$ translate $(\mathrm{mm})$ & 0.18 & 0.35 \\
\hline $\mathrm{y}$ translate $(\mathrm{mm}) \dagger$ & 0.19 & 0.39 \\
\hline z translate $(\mathrm{mm}) \dagger$ & 0.31 & 0.91 \\
\hline x rotate (deg.) $\dagger$ & 0.20 & 0.71 \\
\hline y rotate (deg.) $\dagger$ & 0.20 & 0.48 \\
\hline z rotate (deg.) $\dagger$ & 0.18 & 0.42 \\
\hline
\end{tabular}

(a)

\begin{tabular}{|c|c|c|}
\hline degree of feedom & volunteer & patient \\
\hline$x$ translate $\dagger$ & 24 & 120 \\
\hline$y$ translate $f$ & 39 & 134 \\
\hline $\mathrm{z}$ translate $\dagger$ & 42 & 2350 \\
\hline$x$ rotate $t$ & 85 & 175 \\
\hline y rotate $f$ & 13 & 467 \\
\hline $\mathrm{z}$ rotate & 10 & 257 \\
\hline
\end{tabular}

(b)

Table 1. Median values of (a) range of translational and rotational motion and (b) value of the power spectrum at the stimulation frequency in each degree of freedom for six normal volunteers and eight epileptic patients. †represents significant difference between groups at $95 \%$ level.

The median range of motion and value of the power spectrum for the patient and volunteer groups are compared in table 1. For both indices and all degrees of freedom, the median motion of the patients is greater than for volunteers. The distributions fail a normality test, so the Mann-Whitney non-parametric test was used to test the significance of the difference in these median values. For the range of motion index, the null hypothesis that both groups come from the same population was rejected for all degrees 


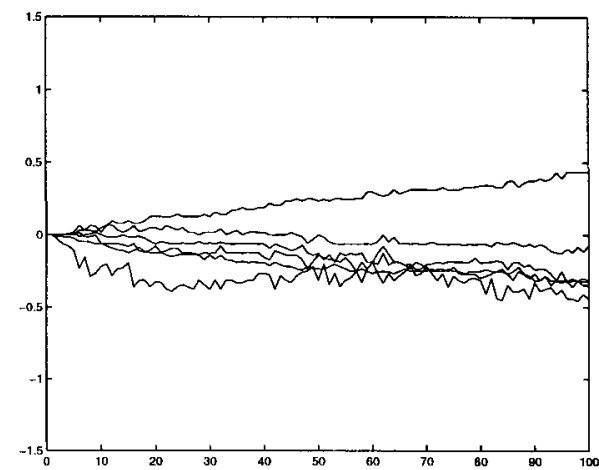

(a)

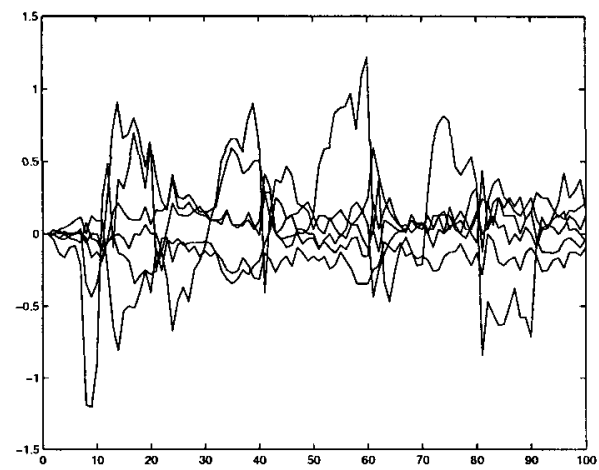

(b)

Fig. 1. Example motion trajectories for a normal volunteer (a) and patient (b). All six degrees of freedom are plotted for each subject. Frame number is along the horizontal axis, and motion amplitude ( $\mathrm{mm}$ for translation and degrees for rotation) on the vertical axes. Note the large amplitude, and more periodic nature of the patient motion.

of freedom at the $95 \%$ level. For the power spectrum measure, the same hypothesis was rejected at the $95 \%$ level for all three translational degrees of freedom and the two rotational degrees of freedom with through-plane component, and at the $90 \%$ level for the in-plane rotational degree of freedom. The degree of freedom of motion that was most significantly greater for the patient group than for the normal group corresponded to through-slice translational motion ( $99.9 \%$ level). The median patient motion when carrying out tasks using the side of the brain affected by the lesion was greater than for the contralateral side for both indices and all degrees of freedom. This data is paired, so the significance of these differences was tested using the paired t-test if a normality test was passed, or Wilcoxon test if the normality test failed. We initially tested for significant difference in motion for each degree of freedom separately. For both motion indices, the motion was significantly greater (at the $95 \%$ level) in the anterio-posterior translation direction (in plane), and for both rotational degrees of freedom with an out-of-plane component. The lack of significant difference for the remaining degrees of freedom may be due to lack of statistical power. We therefore also concatenated the results for all degrees of freedom and carried out a single paired test between the two groups. In this case, for both indices, subject motion when undertaking a motor task on the normal side was significantly less $(99.9 \%$ level) than when carrying out the same task on the side affected by the lesion.

Figure 2 shows axial and coronal slices through a pre-operative MR scan from patient 2 . The CT scan has been thresholded so that only the bone and electrodes are visible and overlaid on the MRI. It is clear that the electrodes lie several millimetres inside the MR brain surface, and this can only be due to brain deformation. The right panel shows combined fMRI and post-implantation CT images from patient 7 , with the significant voxels from the fMRI overlaid as white squares. The electrodes are visible as a row of grey elipses, which, because of brain deformation, lie several $\mathrm{mm}$ inside 

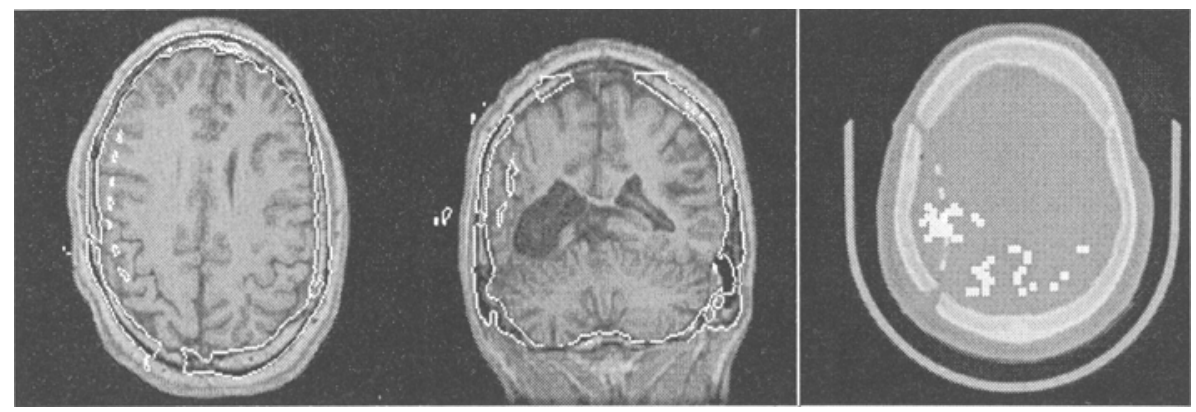

Fig. 2. Thresholded post-implantation CT images overlaid on registered MR image for patient 2 (left panel axial, centre panel coronal). The white lines show the outline of the bone, and the electrodes. Right panel: slice from post-implantation CT scan of patient 7 with significant activated fMRI voxels overlaid in white. The electodes are visible as grey rectangles in the CT. For all patients, there is a considerable gap between the electrodes and inner surface of the skull as a result of brain deformation similar to that shown here.

the surface of the activated cortex. Brain surface deformation has been quantified for the eight patients, and is summarised using the median, 10 percentile and 90 percentile displacements in table 2 .

\begin{tabular}{|l|c|c|c|c|c|c|}
\hline Patient & \multicolumn{3}{|c|}{ median displacement } & \multicolumn{2}{c|}{90 percentile displacement } & \multicolumn{2}{c|}{ 10 percentile displacement } \\
& MR brain & CT skull & MR brain & CT skull & MR brain & CT skull \\
\hline \hline 1 & 3.6 & 4.5 & 7.5 & 7.0 & 1.4 & 0.2 \\
\hline 2 & 4.9 & 5.2 & 9.3 & 9.3 & 2.5 & 2.4 \\
\hline 3 & 3.5 & 3.8 & 7.8 & 6.8 & -2.0 & 1.4 \\
\hline 4 & 4.4 & 5.9 & 10.1 & 10.4 & 0.7 & 1.3 \\
\hline 5 & 10.3 & 7.4 & 13.4 & 11.9 & 6.2 & 1.1 \\
\hline 6 & 6.8 & 5.8 & 10.2 & 10.9 & 2.9 & 4.0 \\
\hline 7 & 3.5 & 5.6 & 9.2 & 13.1 & 0.7 & 2.3 \\
\hline 8 & 5.4 & 7.5 & 11.2 & 12.0 & 0.4 & 1.7 \\
\hline \hline mean (stdev) & $5.3(2.3)$ & $5.7(1.3)$ & $9.8(1.9)$ & $10.2(2.3)$ & $1.6(2.4)$ & $1.8(1.1)$ \\
\hline
\end{tabular}

Table 2. Median, 90 percentile and 10 percentile displacement between the position of the electrodes located in the post-implantation CT scan and the closest point on the brain envelope delineated from the registered pre-operative MR scan (MR brain), and the position of the same electrodes and the closest point on the inner table of the skull (CT skull). Distances are in $\mathrm{mm}$ inside the brain envelope and inner table of the skull respectively.

There is good agreement between the values of the displacements calculated in these two ways. The displacements are all in the same direction, with the electrodes lying inside the brain boundary, as illustrated by figure 2 . The largest shifts, which are more than $1 \mathrm{~cm}$ for most patients, were observed near the centre of the craniotomy, which in many cases is the brain region of greatest interest. These measurements do not provide any estimate of the lateral shift of the brain surface, but provide a lower bound on the deformation of points on the brain surface between the pre-operative MRI and postimplantation CT images. 


\section{Discussion}

In carrying out this study we have identified several sources of error that make it difficult to determine how accurate fMRI is at localizing functional regions, or how well fMRI agrees with invasive electrophysiology measurements.

\subsection{Patient motion during fMRI}

We have found that fMRI is much more unreliable when applied to this group of epilepsy surgery patients than for studies at our centre on normal volunteers. Our results suggest that this is likely to be due to the greater magnitude of motion during scanning of the patient group, and especially the greater magnitude of motion correlated with the stimulus and motion with a large through-plane component.

During fMRI studies, it is necessary to detect changes in image intensity of a few percent [16]. Small amounts of motion can lead to changes of at least this magnitude. For example, consider an imaging sequence in which CSF is $100 \%$ brighter than grey matter. If, between two serial studies, the patient moves laterally by 0.1 of a sampling interval, voxels on the boundary between grey matter and CSF will experience a resulting change in partial volume effect. If the amount of CSF in the voxel increases by $10 \%$, then the intensity of that voxel will increase by $5 \%$. Such changes in partial volume effect at tissue boundaries can either mask signal changes resulting from blood oxygenation effects, or lead to "artefactual activations": signal changes that might be mistakenly interpreted as resulting from functional changes. For properly sampled data, these partial volume changes can be corrected using suitable interpolation kernels. Patient motion within a non-uniform $\mathrm{B}_{0}$ or $\mathrm{B}_{1}$ field can introduce further artefacts, as tissue moving relative to the magnetic field and RF coils will produce signal changes that cannot be corrected for simply by interpolation.

Patient motion that has a component through the slice plane can cause additional problems in multi-slice image sequences. Such through-slice motion can lead to tissue entering the slice that has not previously been excited in the same way as tissue in the slice plane (ie, the tissue has experienced a different "spin excitation history") [17]. Small volumes of particular tissues (eg: grey matter, white matter, CSF) moving into the slice in this way may contribute a different amount of signal to the identical volume of tissue they displace.

If the subject moves with a frequency unrelated to the frequency of the applied stimulus, then this motion can be separated from "true" activation because the period of the activation is known. The patient motion we measured, however, is strongly correlated with the applied stimulus, so its effects cannot be separated from functional effects by their period [18].

\subsection{Brain deformation}

There are numerous sources of error in making the comparison between electrode positions and corresponding positions in the functional MR data. The deformation in the brain surface we have observed for these patients can introduce errors of more than $10 \mathrm{~mm}$ in comparing the location of electrodes to structures and functional regions in 
the pre-operative images. Photographic methods for aligning the location of intraoperatively identified regions or electrodes have been used by other investigators $[4,5]$. These methods are potentially capable of correcting for any rigid body displacement or scale change in the position of the brain. The methods described are, however, unable to correct for deformations of the sort we have observed because they are not simply translational shifts, and the perspective geometry of the intraoperative photographs will introduce further errors that are hard to quantify.

There will be further difficulties in aligning pre-operative functional information from EPI MR images with either intra-operative or post-operative image coordinates because of distortion in the EPI images, which we have not quantified in this study. All these errors taken together make it hard to quantify the degree of agreements between modalities.

\section{Conclusions}

Using current processing techniques, we have found fMRI to be unsatisfactory for localisation of eloquent regions of the cortex in epilepsy neurosurgical patients who have lesions in the vicinity of their sensorimotor cortex. We believe this to be because these patients move more while carrying out motor tasks than other subjects investigated at our centre, and also because this motion tends to have a large component with the stimulus frequency. This is especially true when the subject is carrying out a task using the side of their brain ipsilateral to the lesion. We found substantial deformation in brain surface position between pre-operative MR imaging and post-implantation CT imaging. These brain shifts are of a similar magnitude to the intraoperative shifts recorded by other surgical centres in conventional neurosurgery [19-21]. This, together with possible EPI distortion, suggests that comparisons of the position of regions of activation seen on the fMRI study and the location of electrodes associated with the same functional task are subject to errors that can be in excess of $10 \mathrm{~mm}$. Errors of this magnitude are likely to be clinically significant for many types of surgical procedures, and makes it very difficult to accurately compare the location of electrophysiological measurements with the location of the corresponding blood flow changes measured by fMRI. Methods of comparison involving either post-operative $\mathrm{CT}$, an intraoperative localiser, or intraoperative photographs will all be affected by these errors. Such measurements should, therefore, be interpreted with care, and, using current analysis techniques, are likely to be of limited use in the validation of fMRI protocols. We believe that substantial additional image analysis methodology needs to be developed and applied to these data before accurate comparison of fMRI and invasive electrophysiology can be performed.

\section{References}

1. C. R. Jack, R. Thompson, R. K. Butts, F. W. Sharbrough, P. Kelly, D. P. Hanson, S. J. Riederer, R. Ehman, N. J. Hangiandreou, and G. D. Cascino, "Sensory motor cortex: correlation of presurgical mapping with functional $\mathrm{mr}$ imaging and invasive cortical mapping," Radiology, vol. 190 , pp. 85-92, 1994.

2. T. A. Yousry, U. D. Schmid, A. G. Jassoy, D. Schmidt, W. E. Eisner, H.-J. Reulen, M. F. Feiser, and L. J., "Topography of the cortical motor hand area: prospective study with functional $\mathrm{mr}$ imaging and direct motor mapping at surgery," Radiology, vol. 195, pp. 23-28, 1995. 
3. A. Puce, R. T. Constable, M. L. Luby, G. McCarthy, A. C. Nobre, D. D. Spencer, J. Gore, and T. Allison, "Functional magnetic resonance imaging of sensory and motor cortex: comparison with electrophysiological localization," J. Neurosurg., vol. 83, pp. 262-270, 1995.

4. F. Z. Yetkin, W. M. Mueller, G. L. Morris, T. L. McAuliffe, J. L. Ulmer, R. W. Cox, D. L. Daniels, and V. M. Haughton, "Functional mr activation correlated with intraoperative cortical mapping," Am. J. Neuroradiol., vol. 18, pp. 1311-1315, 1997.

5. D. B. FitzGerald, G. R. Cosgrove, S. Ronner, H. Jiang, B. R. Buchbinder, J. W. Belliveau, B. R. Rosen, and R. R. Benson, "Location of language in the cortex: a comparison between functional mr imaging and electrocortical stimulation," Am. J. Neuroradiol., vol. 18, pp. 1529$1539,1997$.

6. A. Simmons, E. Moore, and S. C. R. Williams, "Automated quality control for functional MRI studies," Neuroimage, vol. 5, p. S466, 1997.

7. D. L. G. Hill, D. J. Hawkes, C. Studholme, P. E. Summers, and M. G. Taylor, "Accurate registration and transformation of temporal image sequences," Proc. Soc. Magn. Reson. 2nd Annual meeting, p. 820, 1994.

8. E. Bullmore, M. Brammer, S. C. Williams, S. Rabe-Hesketh, N. Janot, A. David, J. Mellers, R. Howard, and P. Sham, "Statistical methods of estimation and inference for functional mr image analysis," Magn. Reson. Med., pp. 261-277, 1996.

9. C. Studholme, D. L. G. Hill, and D. J. Hawkes, "Automated 3D registration of MR and CT images of the head," Med. Image Anal., vol. 1, pp. 163-175, 1996.

10. J. West, J. M. Fitzpatrick, M. Y. Wang, B. M. Dawant, C. R. Maurer, Jr., R. M. Kessler, R. J. Maciunas, C. Barillot, D. Lemoine, A. Collignon, F. Maes, et al., "Comparison and evaluation of retrospective intermodality image registration techniques," J. Comput. Assist. Tomogr., vol. 21, pp. 554-566, 1997.

11. J. M. Fitzpatrick, D. L. G. Hill, Y. Shyr, J. West, C. Studholme, and C. R. Maurer, Jr., "Visual assessment of the accuracy of retrospective registration of brain images," IEEE Trans. Med. Imaging, 1998 (in press).

12. J.-D. Boissonnat, "Shape reconstruction from planar cross-sections," Comput. Vision Graph. Image Processing, vol. 44, pp. 1-29, 1988.

13. A. Sahraie, L. Weiskrantz, J. L. Barbur, A. Simmons, S. C. Williams, and M. J. Brammer, "Pattern of neuronal activity associated with conscious and unconscious processing of visual signals," Proceedings of the National Academy of Sciences of the United States of America, vol. 94, pp. 9406-9411, 1997.

14. M. L. Phillips, A. W. Young, C. Senior, M. Brammer, C. Andrew, A. J. Calder, E. T. Bullmore, D. I. Perrett, D. Rowland, S. C. Williams, J. A. Gray, and A. S. David, "A specific neural substrate for perceiving facial expressions of disgust," Nature, vol. 389(6650), pp. 495-498, 1997.

15. G. A. Calvert, E. T. Bullmore, M. J. Brammer, R. Campell, S. C. Williams, P. K. McGuire, P. W. Woodruff, S. D. Iversen, and A. S. David, "Activation of auditory cortex during silent lipreading," Science, vol. 276(5312), pp. 593-596, 1997.

16. S. Ogawa, R. S. Menon, D. W. Tank, S. G. Kim, H. Merkle, J. M. Ellermann, and K. Ugurbil, "Functional brain mapping by blood oxygenation level-dependent contrast magnetic resonance imaging. a comparison of signal characteristics with a biophysical model," Biophysical Journal, vol. 64, pp. 803-812, 1993.

17. K. J. Friston, S. Williams, R. Howard, R. S. Frackowiak, and R. Turner, "Movement-related effects in fmri time-series," Magn. Reson. Med., vol. 33, pp. 346-366, 1996.

18. J. V. Hajnal, R. Myers, A. Oatridge, J. E. Schwieso, I. R. Young, and G. M. Bydder, "Artifacts due to stimulus correlated motion in functional imaging of the brain," Magn. Reson. Med., vol. 31, pp. 283-291, 1994.

19. D. L. G. Hill, C. R. Maurer, Jr., R. J. Maciunas, J. A. Barwise, J. M. Fitzpatrick, and M. Y. Wang, "Measurement of intraoperative brain surface deformation under a craniotomy," Neurosurgery, 1998 (in press).

20. N. L. Dorward, O. Alberti, B. Velani, F. A. Gerritsen, W. F. J. Harkness, N. D. Kitchen, and D. G. T. Thomas, "Postimaging brain distortion: Magnitude, correlates, and impact on neuronavigation," J. Neurosurg., vol. 88, pp. 656-662, 1998.

21. R. D. Bucholz, D. D. Yeh, J. Trobaugh, L. L. McDurmont, C. D. Sturm, C. Baumann, J. M. Henderson, A. Levy, and P. Kessman, "The correction of stereotactic inaccuracy caused by brain shift using an intraoperative ultrasound device," in CVRMed-MRCAS '97 (J. Troccaz, E. Grimson, and R. Mösges, eds.), pp. 459-466, Berlin: Springer-Verlag, 1997. 